\title{
A Direct Method to Estimate Magnetic Helicity in Magnetic Clouds
}

\author{
S. Dasso ${ }^{1,2}$, C.H. Mandrini ${ }^{1}$, A.M. Gulisano ${ }^{1}$ and P. Démoulin ${ }^{3}$ \\ ${ }^{1}$ Instituto de Astronomía y Física del Espacio, IAFE (CONICET-UBA), Buenos Aires, \\ Argentina. email: dasso@df.uba.ar \\ ${ }^{2}$ Departamento de Física, Facultad de Ciencias Exactas y Naturales, Universidad de Buenos \\ Aires, Buenos Aires, Argentina. \\ ${ }^{3}$ Observatoire de Paris, LESIA, UMR 8109 (CNRS), F-92195 Meudon Cedex, France.
}

\begin{abstract}
Magnetic clouds are extended and magnetized plasma structures that travel from the Sun toward the outer heliosphere, carrying an important amount of magnetic helicity. The magnetic helicity quantifies several aspects of a given magnetic structure, such as the twist, kink, and the number of knots between magnetic field lines, the linking between magnetic flux tubes, etc. Since the helicity is practically conserved in the solar atmosphere and the heliosphere, it is a useful quantity to compare the physical properties of magnetic clouds to those of their solar source regions. In this work we describe a method that, assuming a cylindrical geometry for the magnetic cloud structures, allows us to calculate their helicity (per unit length) content directly from the observed magnetic field values. We apply the method to a set of 20 magnetic clouds observed by the WIND spacecraft. To test its reliability we compare our results with the helicity computed using a linear force-free field model under cylindrical geometry (i.e. Lundquist's solution).
\end{abstract}

Keywords. Sun: coronal mass ejections (CMEs), magnetic fields, solar wind, interplanetary medium

\section{Introduction}

Coronal mass ejections (CMEs) are huge expulsions of mass and magnetic field from the Sun. One of the most important roles of CMEs is to carry away magnetic helicity (MH) from the Sun [Low, 1996], that would accumulate incessantly in the active region oroma, since it is generated by the solar dynamo (helical turbulence and differential rotation) without changing sign with the cycle (for a recent review about chirality of magnetic features see Pevtsov and Balasubramaniam [2003]). Since magnetic helicity is well preserved even in non-ideal MHD on a time-scale less than the global diffusion timescale [Berger, 1984], we expect to be able to trace the helicity from the time a flux tube emerges through the photosphere into the corona and is ejected into the interplanetary space, reaching the Earth in a magnetic cloud (MC).

A magnetic cloud can be distinguished [see e.g., Burlaga, 1995], from in situ observations in the interplanetary space, by a low proton temperature, an enhanced magnetic field strength with respect to ambient values, and a large rotation of the magnetic field vector, indicating a helical (flux rope) magnetic structure, which clearly has non-zero helicity.

In order to estimate how much helicity is transported in magnetic clouds it is necessary to determine the size and the global magnetic configuration of these astrophysical objects. The first attempt to estimate the magnetic helicity of MCs was made by DeVore [2000], who used a sample of 18 MCs analyzed by Lepping et al. [1990] using the classical Lundquist's (1950) model. He obtained a mean helicity value of $2 \times 10^{42} \mathrm{Mx}^{2}$ (for a flux 
rope length of $0.5 \mathrm{AU}$ ) and a mean magnetic flux of $1 \times 10^{21} \mathrm{Mx}$ for these MCs. Démoulin et al. [2002] and Green et al. [2002] developed a method to measure the helicity content of active regions in the corona obtaining a typical value of $4-23 \times 10^{42} \mathrm{Mx}^{2}$. Using the same method as DeVore [2000] for the estimation of the helicity content in clouds, they computed the helicity budget for two active regions (ARs) and estimated the amount of helicity carried away by the CMEs ejected from those ARs. However, they did not link the CMEs to any MC observation [see also Mandrini et al. 2004].

Magnetic clouds can be modeled locally using a helical cylindrical geometry as a first approximation [Farrugia et al. 1995]. One of the most commonly models used to describe their magnetic configuration is the linear force-free field [e.g., Lepping et al., 1990]. However, several modeling and fitting methods have been used to reproduce the magnetic structure of MCs [see, e.g., Dasso et al., 2005].

In this paper we present a new method to estimate the magnetic helicity of interplanetary cylindrical flux ropes. We present preliminary results of the application of this method to a set of 20 magnetic clouds. We also compare our results with the values of the helicity per unit length obtained under the assumption of a linear force-free cylindrical model (Lundquist's model, [Lundquist, 1950]) to the magnetic configuration of the cloud. In Section 2 we describe the analysis of the data and our results, while in Section 3, we present our conclusions.

\section{Data Analysis and Modeling}

We select all the MCs observed by the spacecraft Wind from 22-Aug-1995 to 07-Nov1997, taking the start and the end times given in http://lepmfi.gsfc.nasa.gov/mfi/mag cloud_pub1.html. We analyze the magnetic data measured by the Magnetic Field Instrument (MFI) aboard Wind in GSE (Geocentric Solar Ecliptic) coordinates. These observations have been downloaded with a temporal cadence of 3 seconds from http://cdaweb. gsfc.nasa.gov/cdaweb/istp_public/. Because we are only interested in the large scale magnetic structure of the clouds, and not in the magnetic fluctuations, we analyzed smoothed data, only $\sim 100$ averaged points per cloud.

The orientation of the axis of every cloud is obtained using a minimum variance (MV) analysis, as discussed in Bothmer \& Schwenn [1998]. From this analysis we define a system of reference fixed to the cloud and we rotate the observed GSE components of the field to this frame. The cloud frame is defined such that $\hat{x}_{\text {cloud }}$ corresponds to the cylindrical radial direction $(\hat{r})$ in the ideal case of the spacecraft crossing the axis of the cloud (i.e. a null impact parameter, being the impact parameter, $p$, the minimum distance between the cloud axis and the spacecraft) as it leaves the structure, $\hat{z}_{\text {cloud }}$ is parallel to the axis of the cylinder ( $\operatorname{sign}$ such that $B_{z, \text { cloud }}$ is positive at the cloud axis), and $\hat{y}_{\text {cloud }}$ completes a right handed reference system. We also determine the sign of the helicity from the global behavior of the field components. The radius $(R)$ of the cloud is estimated from the duration of the MC and the observed solar wind speed. The list of the start and end times, radius $(R)$, and the helicity sign, are given in Table 1 for the analyzed clouds.

\subsection{Magnetic flux and helicity from observations}

A gauge-independent relative magnetic helicity per unit length $\left(H_{R} / L\right)$ can be defined for cylindrical flux ropes, independently from the reference field [Démoulin et al., 2002], as:

$$
H_{r} / L=4 \pi \int_{0}^{R} A_{\varphi} B_{\varphi} r d r
$$


Table 1. List of the studied magnetic clouds. The start and the end times, the radius $(R)$ of the cloud, and the helicity sign, are shown.

\begin{tabular}{|c|c|c|c|c|}
\hline Event & start & End & $R\left(10^{-2} A U\right)$ & Helicity Sign \\
\hline 1 & 22-Aug-1995 22:00:00 & 23-Aug-1995 19:00:00 & 9.1 & + \\
\hline 2 & 18-Oct-1995 19:00:00 & 20-Oct-1995 00:00:00 & 13.7 & + \\
\hline 3 & 16-Dec-1995 05:00:00 & 16-Dec-1995 22:00:00 & 6.5 & - \\
\hline 4 & 27-May-1996 15:00:00 & 29-May-1996 07:00:00 & 13.2 & - \\
\hline 5 & 01-Jul-1996 17:00:00 & 02-Jul-1996 09:00:00 & 6.5 & - \\
\hline 6 & 07-Aug-1996 13:00:00 & 08-Aug-1996 10:00:00 & 8.6 & + \\
\hline 7 & 24-Dec-1996 03:00:00 & 25-Dec-1996 10:00:00 & 13.0 & + \\
\hline 8 & 10-Jan-1997 05:00:00 & 11-Jan-1997 02:00:00 & 10.1 & + \\
\hline 9 & 21-Apr-1997 15:00:00 & 23-Apr-1997 07:00:00 & 8.9 & + \\
\hline 10 & 15-Мay-1997 09:00:00 & 16-May-1997 01:00:00 & 8.4 & - \\
\hline 11 & 16-May-1997 07:00:00 & 16-May-1997 14:00:00 & 3.6 & - \\
\hline 12 & 09-Jun-1997 02:00:00 & 09-Jun-1997 23:00:00 & 8.2 & + \\
\hline 13 & 19-Jun-1997 05:06:00 & 19-Jun-1997 17:54:00 & 4.7 & + \\
\hline 14 & 15-Jul-1997 06:00:00 & 16-Jul-1997 01:00:00 & 8.2 & - \\
\hline 15 & 03-Aug-1997 14:00:00 & 04-Aug-1997 01:00:00 & 3.2 & - \\
\hline 16 & 18-Sep-1997 00:00:00 & 20-Sep-1997 12:00:00 & 20.5 & + \\
\hline 17 & 21-Sep-1997 22:00:00 & 22-Sep-1997 18:00:00 & 9.9 & - \\
\hline 18 & 01-Oct-1997 16:00:00 & 02-Oct-1997 23:00:00 & 14.8 & - \\
\hline 19 & 10-Oct-1997 23:00:00 & 12-Oct-1997 00:00:00 & 12.0 & + \\
\hline 20 & 07-Nov-1997 05:48:00 & 08-Nov-1997 04:18:00 & 8.4 & + \\
\hline
\end{tabular}

The azimuthal component of the potential vector, $A_{\varphi}(r)$, can be written in function of the partial magnetic flux, $\Phi_{z}(r)$, across a surface perpendicular to the cloud axis as:

$$
A_{\varphi}(r)=\frac{1}{r} \int_{0}^{r} r^{\prime} B_{z}\left(r^{\prime}\right) d r^{\prime}=\frac{\Phi_{z}(r)}{2 \pi r}
$$

and thus, the relative helicity can be computed as an integral of $B_{\varphi}$, weighted with the accumulative flux:

$$
H_{r} / L=2 \int_{0}^{R} B_{\varphi}(r) \Phi_{z}(r) d r .
$$

This expression to compute $H_{r} / L$ is meaningful because it shows that, in this particular structure, the helicity is associated with the azimuthal field surrounding the axial flux. This expression also suggests a method to estimate $H_{r} / L$ directly from the observed field.

For every cloud we construct two subseries for $B_{y, \text { cloud }}$ and $B_{z, \text { cloud }}$. The first subseries corresponds to the data in the period of time when the spacecraft is going into the cloud 

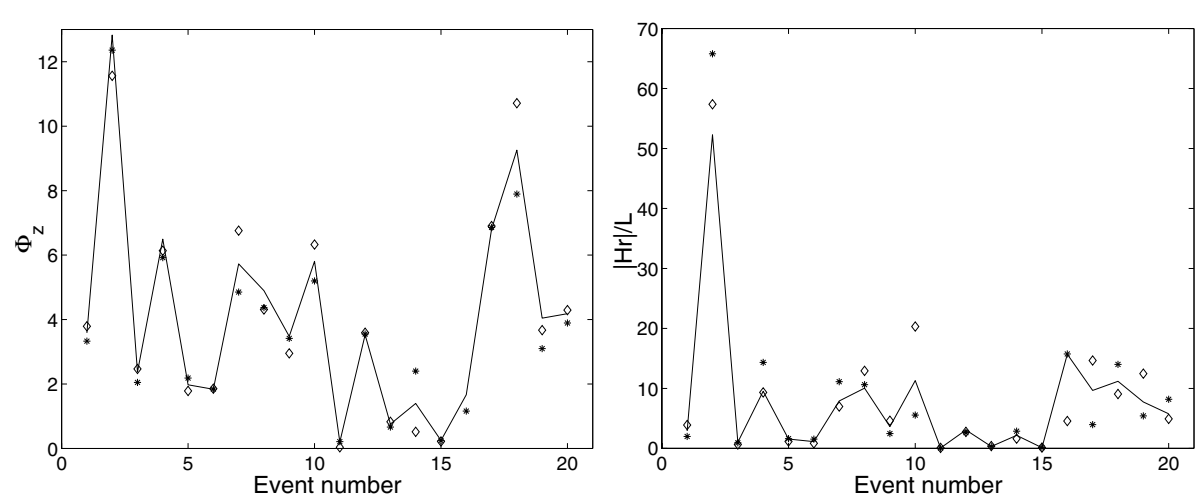

Figure 1. Left panel shows the magnetic flux $\Phi_{z}$, in units of $10^{20} \mathrm{Mx}$, computed from the Lundquist's model (continuous line) and from the direct method (in-bound corresponds to diamonds and out-bound to stars). Right panel shows the absolute values of the relative magnetic helicity per unit length $\left(\left|H_{r}\right| / L\right)$, in units of $10^{41} \mathrm{Mx}^{2} / \mathrm{AU}$, with the same convention as in the left panel.

until it reaches the minimum distance to the cloud axis (in-bound), and the second one from then, during the outgoing travel (out-bound).

Thus, under the assumption of a cylindrical geometry for the cloud and $p \sim 0$, we calculate $\Phi_{z}(r)$ and then, using Eq. 2.3, we compute $H_{r} / L$ for the set of analyzed clouds.

\subsection{Comparison of the direct method with Lundquist's model}

We model the magnetic field configuration of every cloud using the Lundquist's model in the MV coordinates. The physical parameters that fit best the observations $\left(B_{y, \text { cloud }}\right.$ and $\left.B_{z, \text { cloud }}\right)$, and the flux and helicity, are computed following the method described in Dasso et al. [2003].

Figure 1 shows $\Phi_{z}$ and $\left|H_{r}\right| / L$ computed from the direct method and from the Lundquist's model (L). The left panel of this figure shows that the values of $\Phi_{z, L}$ are between the two values computed for each of the two branches (in-bound and out-bound) of the direct method in $14 / 20$ cases. The values of $\Phi_{z, L}$ were the largest in $6 / 20$ events, but for any of the analyzed clouds $\Phi_{z, L}$ was the lowest. The right panel of Figure 1 shows the absolute value of the relative magnetic helicity per unit length $\left(\left|H_{r}\right| / L\right)$. The values of $\left|H_{r, L} / L\right|$ are between those obtained from the two branches of the direct method in $18 / 20$ clouds, and there was no cloud where $\left|H_{r, L} / L\right|$ resulted the largest.

In order to estimate the in-bound/out-bound asymmetry of the clouds, we define $\Delta \Phi_{z}=\left|\Phi_{z, \text { out }}-\Phi_{z, \text { in }}\right|,<\Phi_{z}>=\left(\Phi_{z, \text { out }}+\Phi_{z, \text { in }}\right) / 2, \Delta H=\left|H_{r, \text { out }}-H_{r, \text { in }}\right|$, and $<H>=\left(H_{r, \text { out }}+H_{r, \text { in }}\right) / 2$. Figure 2 shows two histograms for $\Delta \Phi_{z} /\left\langle\Phi_{z}\right\rangle$ and $\Delta H /<H>$, respectively. It can be seen that $\Delta \Phi_{z} /<\Phi_{z}>(\Delta H /<H>)$ is lower than 0.5 in $17 / 20(11 / 20)$ clouds. This means that more than the $50 \%$ of the studied clouds had $\Delta \Phi_{z}$ lower than 0.5 times $\left\langle\Phi_{z}\right\rangle$ and $\Delta H$ lower than 0.5 times $\langle H\rangle$. Thus, we found that the global values of $\Phi_{z}$ and $\left|H_{r}\right| / L$ are affected by the in-bound/out-bound asymmetry in less than the $50 \%$ of their mean values for more than half of the studied MCs.

\section{Conclusions}

We have shown a method to compute the flux and the relative magnetic helicity per unit length for cylindrical flux ropes, and we have applied it to a set of 20 magnetic 

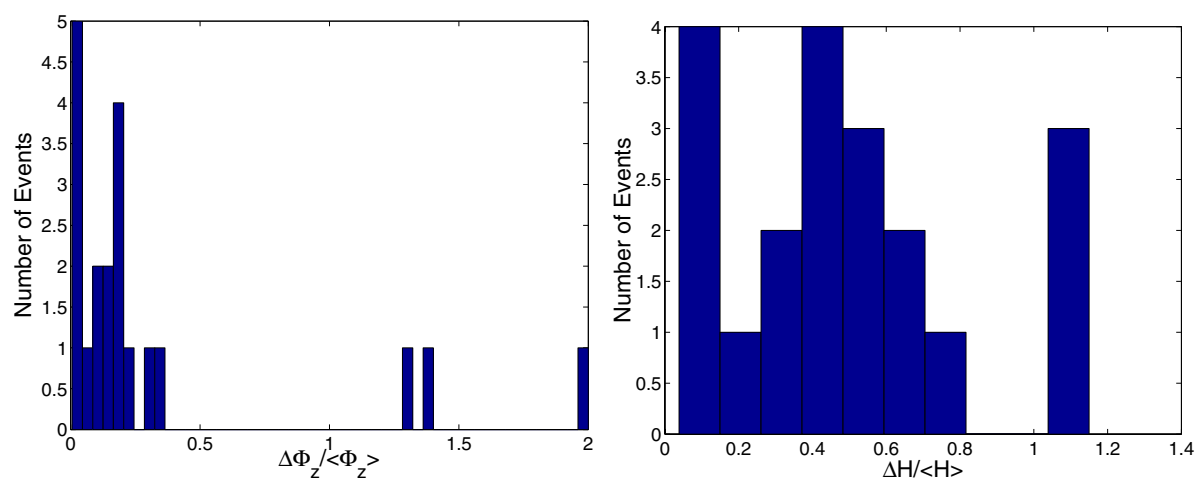

Figure 2. Histogram of the absolute value of the relative difference between in-bound/out-bound flux $\Phi_{z}$ (left panel) and relative helicity per unit length $H_{r} / L$ (right panel).

clouds. We compare the results obtained with the new method to the values derived using the Lundquist's model.

Our results indicate that there is a relatively good agreement between the two ways to compute these quantities, and also that Lundquist's model tends to overestimate the flux and underestimate the helicity in a few cases.

From the three computed values (direct method, in-bound and out-bound, and Lundquist's model) for this particular set of clouds, we find that: $\Phi_{z} \sim 10^{18}-10^{21} \mathrm{Mx}$, with a mean value of $\Phi_{z}=4 \times 10^{20} \mathrm{Mx}$, and $\left|H_{r}\right| / L \sim 10^{39}-10^{42} \mathrm{Mx}^{2} / \mathrm{AU}$, with a mean value of $\left|H_{r}\right| / L=8.2 \times 10^{41} \mathrm{Mx}^{2} / \mathrm{AU}$.

Furthermore, the relative difference between the in-bound and out-bound estimations for the flux and helicity are lower than 0.5 for more than half of our set of clouds. Thus, from our study, $\Phi_{z}$ and $\left|H_{r}\right| / L$ resulted global magnitudes with a relative uncertainty (due to the in-bound/out-bound asymmetry) lower than $50 \%$ for the majority of the clouds.

\section{Acknowledgements}

We thank the NASA's Space Physics Data Facility (SPDF) for their data. This work was partially supported by the Argentinean grants: UBACyT X329, PIP 2693 and PIP 2388 (CONICET), and PICT 12187 (ANPCyT). C.H.M. and P.D. thank ECOS (France) and SECyT (Argentina) for their cooperative science program (A01U04). C.H.M. and S.D. are members of the Carrera del Investigador Científico, CONICET.

\section{References}

Berger, M.A. 1984. Geophys. and Astrophys. Fluid Dyn. 30, 79.

Bothmer, V. \& Schwenn, R. 1998. Annales of Geophys. 16, 1.

Burlaga, L.F. 1995. Interplanetary magnetohydrodynamics, New York, Oxford University Press.

Farrugia, C.J., et al. 1995. J. Geophys. Res. 100, 12293.

Green, L.M., et al. 2002. Solar Physics 208, 43.

Dasso, S., et al. 2003. J. Geophys. Res. 108, 1362, doi:10.1029/2003JA009942.

Dasso S., et al. 2005. Adv. in Space Res., in press.

DeVore, C.R., 2000. Astrophysical Journal 539, 944.

Démoulin P., et al. 2002. Astronomy and Astrophysics 382, 650.

Lepping, R.P., et al. 1990. J. Geophys. Res. 95, 11957.

Low, B.C. 1996. Solar Physics 167, 217. 
Lundquist, S. 1950. Magnetohydrostatic Fields. Ark. Fys. 2, 361.

Mandrini, C.H., et al. 2004. Astrophys. and Space Sci. 290, 319.

Pevtsov, A.A. \& Balasubramaniam, K.S. 2003. Advances in Space Research 32 (10), 1867.

\section{Discussion}

Bothmer: The calculation of helicity is improper and the problems are not named! 1. Compressional effects on B. 2. Application of Bessel-functions. 3. Identification of cloud set. 4. Overall topology and improper references.

DASso: - We presented a method to estimate the content of relative helicity per unit length in the cross section slice of MCs observed at 1AU. Our method assumes a cylindrical symmetry for that cross section, i.e., $\vec{B}=B_{\phi}(R) \hat{\phi}+B_{z}(R) \hat{z}$, and we do not consider expansion or compression effects. Under these assumptions, the relative helicity per unit length that we compute from the observations is properly defined and it is gauge and ideal MHD invariant [see, e.g., Berger \& Field 1984, Demoulin et al, 2002, Dasso et al, 2003]. We compared our new method with the classical Lundquist's solution, using the Bessel's functions in the same way that they were used for modeling MCs in several previous papers [see, e.g., Burlaga, JGR, Q3, 7217, 1988; Lepping et al. 1990; Lepping et al. JGR, 102, 1404Q, 1997]. In this work we analyzed a set of 20 MCs from the Lepping's list (available at http:// Lepmfi.gsfc.nasa.gov/mfi/mag_cloud_pub1.htm). As mentioned in the last viewgraph shown (Summary and Conclusions), we propose to improve our method using expansion effects, elliptical shapes, and comparison with numerical simulations. 